\title{
Multilinguales
}

\section{Contexte et production de sens dans ombre sultane d'Assia Djebar}

Context and Production of Sense in Assia Djebar's Shadow Sultana

\section{Eldjemhouria Slimani}

\section{(2) OpenEdition}

\section{Journals}

\section{Édition électronique}

URL : http://journals.openedition.org/multilinguales/2633

DOI : $10.4000 /$ multilinguales. 2633

ISSN : 2335-1853

\section{Éditeur}

Université Abderrahmane Mira - Bejaia

\section{Édition imprimée}

Date de publication : 1 décembre 2013

Pagination : 139-153

ISSN : 2335-1535

\section{Référence électronique}

Eldjemhouria Slimani, «Contexte et production de sens dans ombre sultane d'Assia Djebar », Multilinguales [En ligne], 2 | 2013, mis en ligne le 01 décembre 2013, consulté le 17 septembre 2019 URL : http://journals.openedition.org/multilinguales/2633; DOI : 10.4000/multilinguales.2633

Ce document a été généré automatiquement le 17 septembre 2019.

\section{(†)

Multilinguales est mise à disposition selon les termes de la Licence Creative Commons Attribution Pas d'Utilisation Commerciale - Pas de Modification 4.0 International 


\title{
Contexte et production de sens dans ombre sultane d'Assia Djebar
}

Context and Production of Sense in Assia Djebar's Shadow Sultana

\author{
Eldjemhouria Slimani
}

1 En règle générale, penser l'enseignement de la littérature c'est penser la transmission d'une culture, d'un patrimoine, d'une histoire littéraire. Pour développer la compétence à la fois lectorale et «culturelle " ${ }^{73}$, il est nécessaire de proposer, à la compréhension, les textes les plus variés afin de familiariser, d'initier progressivement les apprenants à l'appréhension des rapports que le discours entretient avec le contexte pour construire et produire du sens.

2 Les analyses linguistiques et psycholinguistiques de la lecture ont mis en évidence le fait que celle-ci ne se réduit pas à un processus linéaire de constatation d'une signification, mais qu'elle engage un processus dynamique de construction du sens.

3 Ainsi, quelle que soit l'approche méthodologique adoptée pour aborder l'analyse d'un texte, dans le cadre de l'apprentissage d'une langue, une contextualisation s'avère indispensable. Aussi est-il important de clarifier au préalable ces notions de texte, de co-texte et de contexte, pour cerner les rôles et les relations qu'elles entretiennent dans la production et la réception de tout énoncé. C'est ce que nous nous proposons d'étudier à travers Ombre sultane d'Assia Djebar (1987).

4 Nous nous appuierons, au niveau théorique, sur l'apport de la sociocritique qui examine le texte en rapport avec la société et les autres textes (intertextualité), sans négliger l'apport de la linguistique, notamment la théorie de l'énonciation appliquée aux textes littéraires. Comme le précise Dominique Maingueneau :

L'approche strictement "grammaticale" ne peut plus suffire: l'analyste est désormais contraint de s'appuyer sur une théorie de l'énonciation littéraire dont les catégories ne sont réductibles ni à celles de la grammaire, ni à celles de la rhétorique traditionnelle. (Maingueneau, 2003 : p. 6)

5 Il faut, dans un premier temps, déterminer ce que l'on entend par texte. Pour Gérard Genette : 
La critique n'a peut-être rien fait, ne peut rien faire tant qu'elle n'a pas décidé avec ce que cette décision implique - de considérer toute œuvre ou toute partie d'œuvre littéraire d'abord comme texte, c'est-à-dire comme un tissu de figures où le temps (ou comme on dit, la vie) de l'écrivain et celui (celle) du lecteur lisant se nouent et se retordent dans le milieu paradoxal du volume. (Genette, Figures II, 1976 : p. 17)

De fait, notre réflexion sur la littérature s'efforce de prendre en compte deux réalités : le texte et celui qui est à son origine, l'écrivain. Notre proposition consiste à étudier le texte littéraire en ce qu'il implique la prise en compte de la réalité du texte, la réalité de l'écrivain et les conditions socio-historiques de production de l'œuvre.

Notre choix s'est focalisé sur l'étude des exergues, des titres et de l'illustration de la première de couverture d'Ombre sultane d'Assia Djebar, pour tenter d'expliciter les notions de co-texte, de texte et de contexte. Ces composantes sont importantes car elles déterminent en grande partie le choix de l'ouvrage, la lecture et les attentes du lecteur, un (des) sens étant induit(s) dès le titre, l'illustration de couverture, l'exergue ou l'avertissement. Dans l'introduction de Seuils (1987), Gérard Genette investit de « valeur paratextuelle des éléments non linguistiques, qui appartiennent à d'autres systèmes, par exemple iconique : les illustrations, ou matériels : la typographie » (op.cit. : p. 12). Ainsi pour Genette, la paratextualité désigne les relations du texte avec le hors-texte du livre luimême: titres, sous-titres, intertitres, préfaces, postfaces, avertissements, notes, épigraphes, illustrations, bande, jaquette, couverture, etc. Il s'agit d'un ensemble hétérogène comprenant l'écrit et l'image présents dans le livre. Ces éléments paratextuels tout à la fois autonomes et associés au « texte » appartiennent au co-texte qui est défini par Claude Duchet et Isabelle Tournier comme étant :

[...] le point de départ de l'activité qui irradie le « texte » lui-même. Par conséquent le co-texte appartient à la fois au texte et à l'espace référenciel (avec un c), c'est-àdire à l'espace des références (mais déjà sélectionnées, distribuées, opératoires), qui est aussi bien celui de la lecture que de l'écriture. Le co-texte est tout ce qui tient au texte, fait corps avec lui, ce qui vient avec lui (quand on lui arrache du sens)."(Didier (dir.), Dictionnaire universel des littératures, tome 3, entrée «Sociocritique», 1994).

Le co-texte est donc indissociable du texte. Ce dernier étant envisagé comme donnée matérielle, il est important de relever le rôle de l'édition qui contextualise le texte au niveau externe (titre, illustration, etc.), et au niveau interne (typographie, ponctuation, etc.), ainsi que le rôle de l'auteur à qui incombe le choix du mode d'énonciation du texte. C'est pourquoi il est fructueux d'étudier l'impact de cet ensemble hétérogène sur la réception de l'œuvre littéraire. En effet, l'analyse de ces éléments du péritexte, que Jean Peytard désigne par les "entailles $»^{74}$ du texte, nous permettra de voir comment l'union texte et co-texte (et donc contexte) est productive au niveau de la construction du sens.

\section{Étude des exergues et des références intertextuelles dans ombre sultane}

Ombre Sultane met en scène deux femmes que tout semble opposer, mais que tout unit parce qu'ayant eu le même époux. C'est un récit duel relatant alternativement le vécu de Hajila, la seconde épouse issue d'un milieu traditionnel et celui d'Isma, la première épouse et narratrice, femme moderne qui a abandonné le foyer conjugal et qui a marié 
son époux à Hajila, afin de s'en libérer. La mise en parallèle de ces deux trajectoires de femmes, nous offre un récit dans lequel les exergues jouent un rôle d'embrayeur d'une narration paradoxale, à la fois rétrospective et prospective. Le roman se compose de trois parties intitulées : la première : «Toute femme s'appelle blessure ", la seconde : «Le saccage de l'aube », et la troisième: «La sultane regarde ». Chacune de ces parties est introduite par un exergue qui pré-oriente notre lecture, car comme l'affirme Henri Mitterrand :

Des segments d'énoncés désignent et commentent l'œuvre; la première page de couverture, qui porte le titre, le nom de l'auteur et de l'éditeur, la bandeannonce [...], le dos de la page de titre qui énumère les œuvres du même auteur. Toutes ces suites de signes forment un énoncé sur le roman, elles programment un comportement de lecture, elles tendent au lecteur un filet sémantique où il viendra se prendre. (Mitterand, 1980 : p. 15)

L'étude des exergues, choisis par l'auteure, comme matériau intertextuel peut donc nous éclairer sur le (s) sens de l'œuvre et expliquer le choix des titres et leur motivation.

\section{Premier exergue}

11 La première partie, intitulée "Toute femme s'appelle blessure ", est introduite par un exergue extrait de l'œuvre de Germaine Tillion, Le Harem et les cousins (1966) :

Autour de la maison: des murs hauts sans fenêtres, hérissés de tessons de bouteilles; autour du village : toutes les défenses naturelles, les fossés, les figuiers de barbarie; autour de la tente: une horde de chiens à demi sauvages, mais plus sauvage encore que les chiens, une sacralisation de l'espace qui la protège et dont l'inviolabilité se confond avec l'honneur : la Horma. (Djebar, 2006 : p. 13)

Il s'agit ici de la description d'un espace, celui réservé aux femmes, qu'elles soient citadines ou rurales, sédentaires ou nomades. Nous pouvons représenter schématiquement cet espace sacralisé sous forme de cercles concentriques qui symbolisent :

- la maison aux façades aveugles et cernée de remparts de toutes sortes chez les citadins ou les sédentaires, et

- la tente entourée de défenses naturelles et sur laquelle veille une horde de chiens chez les bédouins ou les nomades :

\section{La maison}

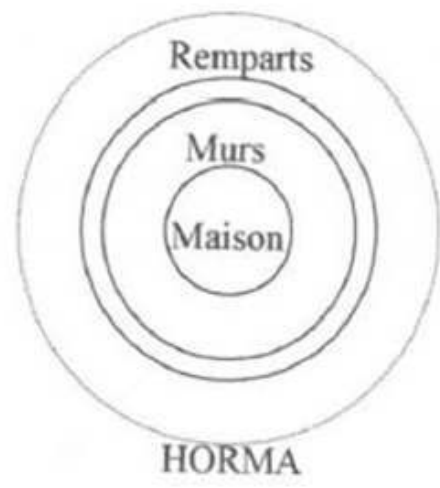

\section{La tente}

ou

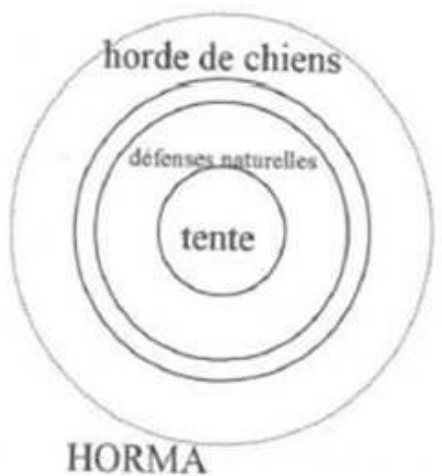


13 Il s'agit d'un espace défensif, défendu, inviolable, car la maison se confond avec le harem puisque c'est le territoire réservé à la femme. C'est donc un espace protégé, dont l'accès (pour un étranger) est réglementé (voire interdit) de façon très stricte. C'est enfin l'espace où l'homme règne en "maître» sur les femmes. En outre, l'espace féminin est un espace sacré, et c'est en tant que tel qu'il est ancré dans la conscience collective arabe visée par le texte: "c'est dans la parenté féminine d'un homme que l'on pouvait atteindre le plus profondément son honneur. " (D'Huart, Tazi, Harems, 1980, p. 10). Si l'on se réfère à l'ordre social traditionnel de la société algérienne pendant la colonisation et qui a prévalu même au lendemain de l'indépendance (et qui prévaut encore dans plusieurs régions), les femmes ne sortaient pas à l'extérieur du foyer (sauf pour des déplacements ritualisés : visites de Saints, bain maure, etc.). C'est cette idée que reprend Assia Djebar dans L'amour, la fantasia (1985), par exemple :

Jamais le harem, qu'il soit d'habitation ou de symbole parce qu'il empêcha le métissage de deux mondes opposés, jamais le harem ne joua mieux son rôle de garde-fou: comme si les miens déracinés, comme si mes frères et par là mes geôliers avaient risqué une perte de leur identité. (p.145)

14 Pour cet auteure, c'est l'espace de la claustration, l'espace de l'ombre, de l'ensevelissement, des interdits qui affectent le corps féminin et qui sont désignés par le terme même de "harem " (ou "péché » au sens strictement religieux). La mise en exergue de cet espace de confinement est en fait mise en scène du sort fait aux femmes. Corps voilé, momifié, interdit de regard, interdit de parole et regard réprobateur de la société sur toute femme qui ose braver l'interdit. Ce qui peut expliquer le choix du titre "Toute femme s'appelle blessure». Cet espace est également mis en texte d'une revendication féministe : comment se libérer du joug d'une société patriarcale ? Tout simplement en franchissant dans un premier temps le "Seuil», ce qu'Assia Djebar appelle « la rupture» (p.10) qui est assimilée à la prise de conscience de la femme qui «sort», signifiant par cet acte la prise en charge de sa propre personne au niveau physique et de sa destinée.

Simone Rezzoug rapporte, dans sa Monographie sur Assia Djebar, que pour cette auteure : «tous les personnages féminins subissent avec des variantes de peu d'importance, le même destin et vivent dans un semblable malaise leur condition de recluses» (Rezzoug, 1991 : p. 70).

16 Ainsi, Assia Djebar s'inspire d'une réalité sociale, car aujourd'hui encore, sous des apparences d'émancipation, nombreuses sont les femmes qui subissent le diktat du harem, qui a matériellement disparu presque partout mais qui est encore fortement ancré dans les mentalités.

Remettant en cause la structure du harem, Isma s'est libérée de l'homme en utilisant Hajila comme relais. A Hajila qui se trouve confrontée à la même altérité et qui est en rupture potentielle, Isma s'adresse ainsi dans l'incipit : « Nous voici toutes deux en rupture de harem mais à ses pôles extrêmes: toi au soleil désormais exposée, moi tentée de m'enfoncer dans la nuit ressurgie » (p. 10).

18 Pour Isma, éviter le soleil, cela veut dire réduire le conflit frontal avec la société patriarcale. Elle choisit le «clair-obscur », «l'ombre dans laquelle elle a reculé» (p.9-10) par peur de l'éblouissement aveuglant de la lumière.

19 La thématique du regard travaille le texte d'Assia Djebar : regard vide de la momie, regard de morte à mettre en rapport avec le regard absent de soi-même de la femme et le regard nécrophore d'une société sur une réalité féminine particulière. C'est ce qui justifie, de notre point de vue, le choix d'un portrait funéraire féminin ${ }^{75}$ en première de 
couverture de cette œuvre, portrait où l'éclairage se concentre sur les yeux immenses et dont le regard vide reflète un univers de tristesse et d'immobilité.

La mise en rapport de quelques extraits avec l'image de couverture met en relief la vision pessimiste de la narratrice sur ce présent immobile des femmes de sa communauté, sur l'absence de perspectives pour celles qui ont choisi le champ de la transgression, à savoir d'abord "sortir », dévoilées ou non, occuper l'espace du dehors, réservé jusqu'à peu uniquement aux hommes. Pessimisme qui se traduit au niveau du lexique qui relève du champ sémantique de la mort, de la momification :

Tu te réenveloppes du haik. Dehors te revoici fantôme et la colère grisâtre replie ses ailes sous la blancheur du drap. [...] engoncée dans ce linceul, tu te laisses tomber sur le matelas, par terre [...] d'un coup, la laine tombe sur tes hanches, dévoile ton visage. La laine du suaire. (p. 38,39$)$

"Angoisses que les mères vous transmettent, obsessions d'un ailleurs informulé, tout vous est lien, bandelettes et carcan. (p.95) [...] Les matrones emmaillotent leurs fillettes pas encore pubères de leur angoisse insidieuse. (p. 155) [...] Emmaillotées, nous nous présentons au monde fantômes aux yeux ouverts » [...] «Dans ton visage masqué, un seul œil est découvert, la trouée juste nécessaire pour que ce regard d'ensevelie puisse te guider. » (p.164)

21 Il est important de souligner l'importance du thème de la lumière dans Ombre sultane en particulier, et dans la plupart des écrits djebariens en général. S'adressant à Hajila, Isma dit : «le mal a jailli du dehors, de son sourire décapant il t'expulse [...] tu t'es élancée trop avidement, personne ne t'a mise en garde contre le soleil » (p. 98).

L'écriture dans ce roman se déroule sur une double partition métaphorique : Ombre vs lumière / Silence vs parole. Le dehors est associé au soleil, l'intérieur à l'ombre. Le monde du dehors, de la lumière réservé jusqu'à présent aux hommes, s'oppose au monde du dedans, à l'espace féminin ou "harem" associé à l'ombre. À l'ombre murmurante du monde féminin, s'opposent les bruits, « la rumeur menaçante du dehors » (p.10), car l'extérieur, cet espace lumineux, est aussi espace de la parole, de «la vérité ». Le dehors associé à l'aube et donc à la lumière est chargé de menaces pour Hajila en "rupture de harem» (p.10). Isma perçoit "dans chaque passagère le passage de l'ombre au soleil, du silence au mot, de la nuit au nu de la vérité » (p.167).

Assia Djebar s'explique à ce sujet dans une interview au journal français Le Monde du 29/05/1987 :

Mon sens de la solidarité féminine s'exprime ainsi : montrer celles qui franchissent le seuil, montrer les ruptures et évolutions qui se font sans accentuer l'expulsion. Comment écrire, je ne dirai pas cet arrachement, mais ce début d'envol sans se couper du reste.

La liberté de mouvement traduit l'évolution des mentalités. Et c'est dans cette prise de conscience du corps féminin en mouvement qu'Assia Djebar tente de cerner la liberté effective de la femme algérienne. Mais cette liberté est encore précaire car les esprits sont encore " emmaillotés", pris dans le carcan des mentalités rétrogrades. Comment dès lors, libérer la parole féminine? En développant le concept de sororité, comme le préconise la narratrice :

La deuxième épouse refera ce que la première a seulement esquissé ; franchir les mêmes halliers, faire lever, sous l'éclair de diamant de la lucidité, même folie improvisée. La première, guetteuse, furtive attend, entend. L'étape suivante nécessite le halo des mêmes projecteurs -soleil ou chandelle d'alcôve-ré allumés. Alors la première femme va disparaitre, se dissoudre là, renaître ailleurs. La 
seconde femme se présente sur le seuil, avaleuse d'espace ; la première dès lors peut se voiler, ou se dissimuler. (p.169) Isma-Hajila: arabesques de noms entrelacés. Laquelle des deux, ombre, devient sultane, laquelle, sultane des aubes, se dissipe en ombre d'avant midi? » (p.10) et qui renvoie au titre du roman et annonce le second exergue : Hajila et Isma sont tour à tour ombre et sultane car elles échangent leurs places et leurs rôles auprès de l'Homme, et ces deux destins liés explicitent le concept de sororité que l'auteure illustre par la référence à la sultane Schéhérazade qui sans l'aide de Dinarzade, sa sœur, aurait été tuée.

\section{Second exergue}

Schéhérazade dit à sa sœur :

Ma chère sœur, j'ai besoin de votre secours dans une affaire très importante; je vous prie de ne me le pas refuser. Mon père va me conduire chez le sultan pour être son épouse. Que cette nouvelle ne vous épouvante pas ; écoutez-moi seulement avec patience. Dès que je serai devant le sultan, je le supplierai de permettre que vous couchiez dans la chambre nuptiale, afin que je jouisse cette nuit encore de votre compagnie. Si j'obtiens cette grâce, comme je l'espère, souvenez-vous de m'éveiller demain matin, une heure avant le jour. (Djebar, 1987, 2006 : p. 137)

Ce second exergue est un extrait des Mille et une nuits (traduction de Mardrus) et se situe en amont de la première nuit. La référence à Schéhérazade la sultane, qui raconte des récits merveilleux à son époux, afin de reculer le jour où il doit la faire périr, met en évidence la solidarité entre sœurs, entre femmes. Chaque aurore devient menace pour Schéhérazade et c'est grâce à Dirnazade, la sœur, que la conteuse s'éveille à chaque aube, pour raconter au sultan Chahrayar, à l'homme, ces histoires extraordinaires qui lui permettent de différer l'échéance de la mort. Elle renvoie aussi à une réalité politique, historique et culturelle précise à savoir le statut de la femme dans la société arabo-musulmane, patriarcale : femme assujettie, condamnée à vivre dans l'ombre du mâle. C'est ainsi que cet exergue reconduit de manière implicite l'espace du harem. Femme interdite de parole ou plutôt, comme le souligne Hafid Gafaïti, dont le « discours se situe dans les zones d'ombre que sont l'interdit, le tabou, le silence, le refoulement, le drame de la parole qui sont l'essence même de la femme dans la société arabo-musulmane» (Gafaïti, 1996 : p. 215).

Le choix de cet exergue a une valeur symbolique : Schéhérazade est l'exemple même de la femme qui refuse d'être la victime expiatoire du machisme, et qui, pour éviter d'être broyée par la misogynie, a su grâce à son intelligence, grâce au verbe et surtout grâce à l'entraide sororale, échapper à une mort certaine.

La mise en relation de cet exergue avec le titre de la seconde partie, "le saccage de l'aube », et avec le titre du roman, nécessite d'ancrer l'œuvre dans la réalité sociohistorique de l'Algérie. Ombre Sultane, paru en 1987, inscrit le contexte de production de ce roman dans celui de la lutte des femmes contre la régression et la montée de l'intégrisme religieux. L'irruption massive des travailleuses et des étudiantes qui se sont emparées de l'espace public va voir l'émergence de revendications féministes. Soumia Salhi ${ }^{76}$ retrace, dans un article intitulé «Les Algériennes 50 ans après », paru dans le quotidien algérien $E l$ Watan du 08/10/2013, les différentes étapes d'émergence des mouvements féministes:

Adossés aux mouvances de la gauche clandestine, des cercles clandestins apparaissent à Alger, Oran, Constantine et Annaba déjà en 1977. On passe ensuite à 
des activités publiques dont les plus significatives sont les ciné-clubs féminins. En 1980/81, ce sont des collectifs qui se mettent en place. Après ces années de maturation, c'est un vaste mouvement qui prend la parole à partir de 1988.

Sur le plan discursif, Ombre sultane développe deux discours parallèles. Le premier consiste en un discours descriptif de l'oppression des femmes accompagné de sa dénonciation ; le second, qui se veut positif, peut être considéré comme une alternative au premier en développant le principe de "sororité ", et de ce fait, étend le propos à l'universalité. Ce concept de sororité utilisé par Assia Djebar désigne la prise de conscience d'une liberté possible grâce à la naissance d'une solidarité féminine essentielle. L'ouverture du roman s'achève sur cette phrase indicative de la communauté de destin des femmes : «Et notre peur à toutes aujourd'hui se dissipe, puisque la sultane est double » (p.107).

Dans son étude intitulée Les femmes dans le roman algérien, Hafid Gafaïti écrit :

En écrivant le vécu de ses consœurs, la romancière atteint un double but, d'une part les faire entrer dans l'Histoire par la fonction fondatrice de l'écriture; d'autre part, cela lui permet d'établir un pont vers les femmes des autres pays, démontrant, en parlant également leur langage par le biais de la problématique de la sororité récemment mais solidement conceptualisée par les féministes occidentales dont le discours de Djebar est proche, que si leurs situations ne sont pas assimilables, leur destin est commun. Elle atteint ainsi l'objectif de tout écrivain qui partant du singulier tente de se fondre dans l'universel. En tendant la main vers l'autre, elle établit le lien avec toutes les femmes. (Gafaïti, 1996 : p. 227)

31

Ainsi, la convocation intertextuelle des Mille et une nuits et la mise en relation du texte avec les catégories idéologiques de l'Orient permettent à l'écrivain de choisir "l'aire sociale au sein de laquelle il décide de situer la nature de son langage ", selon les termes de Roland Barthes (Barthes: 1972, p.19). En effet, de par sa formation, Assia Djebar, écrivant en français, s'adresse nécessairement au moins à deux publics : celui de son pays, et celui de tous les autres pays qui peuvent la lire, les publics français et francophone en particulier. Or, si Assia Djebar choisit l'intertexte Les Mille et une nuits, c'est parce que c'est le texte oriental le plus marqué et c'est également un texte que l'Occident a assimilé depuis fort longtemps.

La figure de Schéhérazade est familière au lecteur occidental et les catégories de représentation de la femme arabe qu'il véhicule confortent sa propre représentation de celle-ci. Cette référence à l'Orient dans l'œuvre d'Assia Djebar indique le travail de récupération au niveau intertextuel et opère dans le déplacement : un Orient au centre de son propre univers. Aussi, pour cette auteure, le choix de Schéhérazade, mythe littéraire comme origine et référence, est-il une tentative d'identification de la femme «écrivaine » à ce symbole du dire féminin, et le point de départ d'une solidarité se 
transmettant de femme en femme. On peut encore ajouter que le choix de Schéhérazade et de sa sœur Dirnazade comme exergue permet à notre auteure d'illustrer la notion de sororité car, ainsi que l'explique Frédéric Joignot, « c'est pourquoi l'extraordinaire personnage de Schéhérazade continue de séduire les féministes de culture arabe (et pas seulement), qu'elles se réclament de sa parole émancipatrice - comme Assia Djebar » (Le Monde, 29 /12/2012).

De plus, le dire féminin maghrébin relevant essentiellement de l'oralité et voué à disparaître, l'écriture devient pour Assia Djebar le moyen essentiel de pérenniser les voix des femmes. En paraphrasant T.Todorov, qui écrit au sujet des Mille et une nuits, « pour que les personnages puissent exister, ils doivent raconter " (Todorov, $1980:$ p. 89), nous pouvons affirmer que pour que les femmes puissent exister, elles doivent écrire, car « paroles de femmes sont paroles d'ombre ».

C'est l'objectif qu'Assia Djebar, dans une interview accordée à Tahar Djaout, dans le journal algérien Algérie actualités, assigne à son écriture :

Ecrire m'a ramenée au cri des femmes sourdement révolté de mon enfance, à ma seule origine. Ecrire ne tue pas la voix mais la réveille pour ressusciter tant de sœurs disparues...Pour les générations d'Algériens formés avant 1962 - dont je fais partie - l'espace féminin était la grotte fertile où les aïeules de la tribu nourrissaient les mémoires. Rappeler ce dedans où s'entretenait l'histoire orale, c'est pour moi m'adresser à tous ceux de mon pays, hommes et femmes. (Semaine du 29 mars au 04 avril 1990, n 1276)

L'auteure précise quelques années plus tard, dans un entretien accordé à Tahar Benjelloun, dans le journal Le Monde:

Tout le Maghreb a refusé l'écriture. Les femmes n'écrivent pas. Elles brodent, se tatouent, tissent des tapis et se marquent. Ecrire c'est s'exposer. Si la femme, malgré tout, écrit, elle a le statut de danseuses, c'est-à-dire des femmes légères. Une femme algérienne qui se met à écrire risque d'abord l'expulsion. (29 mai 1987)

Ainsi, selon Assia Djebar, une femme qui écrit s'expose à sa dévalorisation sociale et à la vindicte de la société. Mais « le récit de la sultane des aubes » (p.113) sauvera-t- il l'une de ces opprimées? Comment faire naître cette parole étouffée non seulement par le discours dominant du mâle, mais aussi par la langue française dans laquelle elle est contrainte de s'exprimer ? Comment se dire dans la langue de « l'Autre » sans trahir les siens? Comment remplacer l'arabe ou le berbère, langues intimes, matrices des émotions et des sentiments, par le français, langue distante, extérieure ? Telle est le thème récurrent de son écriture que l'on retrouvera tout au long de son œuvre. Ramener au grand jour ce "dedans », parler de cet «espace féminin » dans la langue étrangère, c'est s'exposer doublement. Cette écriture féministe en langue française place en effet notre auteure dans le double champ de la transgression et de l'aliénation. Et tout comme Schéhérazade, la femme écrivaine "se meut entre le trône et l'holocauste " (p. 107) et reste dans l'expectative : "elle regarde » comme l'annonce le titre de la troisième partie et l'exergue qui l'inaugure.

\section{Troisième exergue}

La lune était sereine et jouait

Sur les flots

La fenêtre enfin libre est ouverte à

La brise

La sultane regarde (Assia Djebar, 2006 : p. 201) 

romanesque proprement dit, nous avons tenté de contextualiser cette œuvre, qui se veut témoignage de la place de la femme au sein de la société. La diseuse "regarde ", observe la société. source de renouvellement. En nous référant à la période de parution du quatuor ${ }^{78}$ d'Assia Djebar (1980-1989), de mille neuf cent quatre-vingt à quatre-vingt-neuf (avec Ombre Sultane en1987), nous constatons qu'elle correspond à celle des luttes de femmes sur le plan national et international. Pour elle, les termes déterminant la situation des femmes dans le monde arabo-musulman n'ont pas changé fondamentalement. En effet, dans la foulée du mouvement féministe des années quatre-vingt (1980), notre auteure prend fait et cause pour toutes les femmes de sa société, cloîtrées et emmurées dans le silence. 
formation d'historienne, sa sensibilité de femme et de poète, mais surtout sa maitrise incontestable de la langue française lui permettent de mettre en mots un monde féminin clos, feutré, fait de sensations, de sentiments, et ainsi de développer le concept de sororité notamment dans Ombre sultane.

44
notion de contexte par rapport à un élément d'un texte, car «le contexte est l'ensemble du texte qui entoure cet élément et dont l'assemblage permet de donner tout son sens à un énoncé ». (Grand dictionnaire des Lettres : p. 370).
Soulignons, cependant, que les mérites d'un livre, d'un texte dirons-nous, sont reconnus en fonction de sa qualité littéraire, de la résonance qui nous le rend inoubliable, et ce, indépendamment des différentes sources d'inspiration ou du contexte de production

45

\section{BIBLIOGRAPHIE}

BARTHES, Roland, Le degré zéro de l'écriture, Paris, Seuil, 1972.

CHIKHI Beida, Littérature algérienne, désir d'histoire et esthétique, Paris, éd. L'Harmattan, 1997.

CHIKHI, Beida, les romans d'Assia Djebar, Alger, O.P.U., 1990.

DIDIER, Béatrice, Dictionnaire universel des littératures, vol. 3, PUF, 1994.

DJEBAR, Assia, L'amour, la fantasia, Paris, J.C. Lattès éd., 1985.

DJEBAR, Assia, Ombre sultane, Paris, J.C. Lattès éd., 1987.

DJEBAR, Assia, Ombre sultane, Paris, Albin Michel éd. 2006.

DJEBAR, Assia, Vaste est la prison, Paris, Albin Michel, 1990.

D'HUART, Anabelle, TAZI, Nadia, Harems, Paris, Ed. Chêne, Hachette, 1980.

DUCHET, Claude, TOURNIER, Isabelle, entrée : « Sociocritique », in Dictionnaire universel des littératures, publié sous la direction de Béatrice Didier, vol. 3, PUF, 1994.

GAFAÏTI Hafid, Les femmes dans le roman algérien, Paris, L'Harmattan, 1995.

GENETTE Gérard, Figures II, Paris, Ed. du Seuil, coll. « Tel Quel », 1969, coll. « Points essais », 1976, (notre édition de référence).

GENETTE Gérard, Seuils, Paris, éd. du Seuil, 1987.

MAINGENEAU Dominique, Linguistique pour le texte littéraire, Paris, Nathan, 2003, $4^{\mathrm{e}}$ édition.

MITTERAND Henri, Le discours du roman, Paris, PUF, coll. « Écriture », 1980.

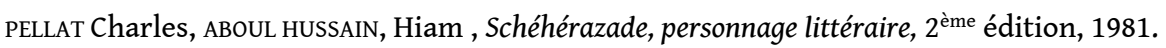

PEYTARD Jean, Littérature et classe de langue, Paris, LAL, Crédif-Hatier, 1982. 
REZzoUG Simone, « Monographie sur Assia Djebar » in C. ACHOUR (dir.), Diwan d'inquiétude et d'espoir, la littérature féminine de langue française, Alger, ENAG, 1991, p. 70.

TILLION Germaine, Le harem et les cousins, Paris, Le Seuil, coll. Points, 1966.

TODOROv Tzvetan, Poétique de la prose, Paris, Le Seuil, 1980.

\section{Articles de presse}

AMMAR KHODJA Soumeya, « ombre sultane, souffrance séculaire », Liberté du 19/01/1991.

BENJELLOUN Tahar, Entretien avec Assia Djebar, « Nos mères n'avaient pas conscience du dehors », Le Monde du 29/05/1987.

BERAH Mounir, « Ombre sultane d'Assia Djebar », Révolution Africaine n 1201 du 06/03/1987.

DJAOUT Tahar, « De la fiction du roman aux images de la réalité », Algérie-Actualité n 1276 semaine du 29 mars au 04 avril 1990.

моUғғок Ghania, « A la recherche de l'aïeule », Algérie-Actualité n 1049 semaine du 21 au 27 novembre 1985 , p. 32.

SALHI Soumia, « Les Algériennes 50 après... », EL Watan du 8 /10/2013, p. 25.

\section{NOTES}

73. Les deux compétences sous-tendent le développement de la compétence scripturale.

74. Jean Peytard: "Le texte étant aussi bien constitué par une citation, un titre de roman, une page prélevée, que par une œuvre complète ", Littérature et classe de langue, Paris, LAL, Crédif-Hatier, 1998, p. 109.

75. Ce portrait a été découvert dans une nécropole du Fayoum en Égypte. Il date du début de l'ère chrétienne et remplace l'ancien masque funéraire de momie.

76. Militante féministe et syndicaliste.

77. Nous précisons qu'il s'agit d'une maison d'édition féministe.

78. Les romans d'Assia Djebar Femmes d'Alger dans leur appartement(1980), L'amour, la fantasia (1985), Ombre sultane (1987) et Vaste est la prison (1995) peuvent être considérés comme constituant un quatuor car la réflexion de l'auteure s'approfondit au fil de ces livres pour rendre compte du vécu réel des femmes de sa société et l'étendre à toutes les femmes arabes. En aiguillant le lecteur sur les problèmes qui ont agité l'âme d'une génération d'Algériens victimes d'une bifurcation de l'histoire et de leur double culture, notre auteure militante féministe souligne combien ils sont vécus plus douloureusement par les femmes.

\section{RÉSUMÉS}

La littérature étant considérée par les didacticiens des langues comme un des véhicules de la culture, nous avons jugé intéressant de contribuer à chercher les méthodes les mieux appropriées pour l'appréhender du point de vue de sa relation à la société. Dans cet ordre d'idées, 
nous croyons que l'entrée dans le texte littéraire par les éléments qui gravitent autour de lui est à même d'orienter le lecteur vers la construction du sens en s'appuyant notamment sur ses connaissances du contexte. Nous nous proposons de montrer, à travers la lecture les exergues d'un roman d'Assia Djebar, comment texte et co-texte sont étroitement imbriqués pour produire du sens, et ce qu'implique la notion de contexte en rapport avec le fait littéraire.

Since literature is considered by language teachers as a vehicle of the foreign culture, it would be interesting to contribute to research on the most appropriate methods to teach it effectively. In the same vein, we believe that entering a literary text through the elements that revolve around it can direct the reader to the construction of meaning based on his/her prior knowledge. Therefore, we propose to show, through the reading of illustration and epigraphs from one of Assia Djebar's novels, how text and co-text are closely interwoven to produce meaning, and thus to define the notion of context related to the literary fact.

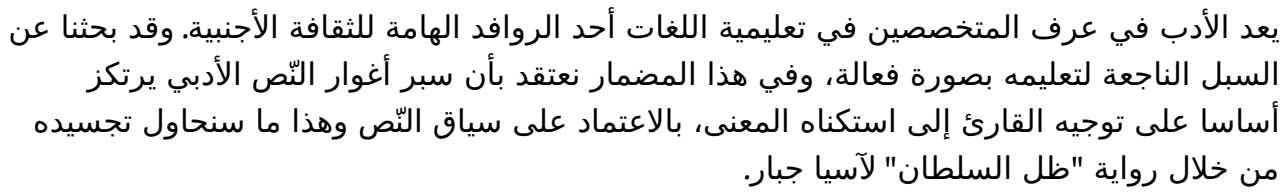

INDEX

$$
\text { النّص, النّص المحيط, التناص, السياق, النّص المصاحبفهرس الكلمات المفتاحية: }
$$

Keywords : text, peritext, intertextuality, context, co-text

Mots-clés : texte, péritexte, intertextualité, contexte, co-texte

\section{AUTEUR}

\section{ELDJEMHOURIA SLIMANI}

Université H. Benbouali - Chlef - Algérie 